\title{
Technical and methodological aspects of PET/MR
}

\author{
Sibylle I. Ziegler · Gaspar Delso
}

Received: 19 December 2012/ Accepted: 1 February 2013/Published online: 22 February 2013

(C) Italian Association of Nuclear Medicine and Molecular Imaging 2013

\begin{abstract}
The combination of PET and MR in a single device can be accomplished by different approaches, but it is technically much more demanding than combining PET with CT. A number of research projects resulted in combined PET/MR systems for preclinical use. Different approaches for clinical systems are described in this overview. These systems bring new challenges in PET quantification.
\end{abstract}

Keywords PET/MR systems - Quantification · MR-compatible PET

\section{Introduction}

The clinical success of positron emission tomography (PET) obviously prompted the idea of combining the functional information from PET with the high soft-tissue contrast from magnetic resonance imaging (MR). For a long time, software-based images of coregistration solutions have bridged the gap between separate scanners. Coregistration of separately acquired data sets may be inaccurate due to patient repositioning and differences in patient support devices in the PET or MR scanner. In addition, identical patient status and clinical condition cannot be guaranteed when scanning the patient occurs with two separate devices. In principle, simultaneous acquisition of PET and MR signals provides perfect spatial

\section{S. I. Ziegler $(\bowtie)$}

Department of Nuclear Medicine, Klinikum rechts der Isar,

Technische Universität München, Munich, Germany

e-mail: sibylle.ziegler@tum.de

G. Delso

Klinik für Nuklearmedizin, Universitätsspital Zürich,

Zurich, Switzerland and temporal registration. Thus, from a technical point of view, simultaneous acquisition of PET and MR has clear advantages. But the technical challenges of developing integrated PET/MR instrumentation are much more demanding than in the case of PET/CT.

The first studies on the combination of PET and MR were conducted with the aim of reducing the positron range in high magnetic fields [1]. This aspect has become less important with simultaneous PET/MR measurements since very high magnetic fields are needed to improve resolution for commonly used positron emitters such as F-18 or C-11. Still, even at field strengths of $3 \mathrm{~T}$, the positron range is influenced and at tissue borders artifacts may arise that need to be considered while reading images from combined PET/MR instrumentation. Correction of these effects in the image reconstruction procedure may be feasible but is not yet clinical routine [2].

\section{The technical challenges of PET/MR}

The main problem in combining PET and MR arises from the fact that photomultiplier tubes cannot be operated in a magnetic field without degradation of signal performance. In addition, there is only very limited space if, for a fully integrated system, the PET detector is to be placed in the magnet. Therefore, it is not possible to use the standard PET detector comprising crystal arrays and photomultiplier tubes. Pioneering work in the development of MR-compatible PET detectors has been conducted at UCLA [3]. The detector principle is based on the use of long optical fibers which guide the light from scintillation crystals positioned within the magnetic field to position-sensitive photomultiplier tubes outside where the fringe field drops below $10 \mathrm{mT}$. The length of the light guide was 3-4 m. 
Based on this technology, the first simultaneous PET and MR imaging of phantoms at $1.5 \mathrm{~T}$ was performed with a single-layer LSO ring of $54 \mathrm{~mm}$ diameter [4]. Artifact-free simultaneous PET and MR images could be obtained with this prototype system and various MR scanning protocols [5]. The feasibility of simultaneous PET and MR imaging as well as MR spectroscopy for small animals has been shown using a similar prototype [6].

However, poor overall performance of the PET device was noticed [6], the main reasons for this being the limited axial extent, low sensitivity and reduced PET performance caused by the long light guides. Also, if a larger axial volume is to be covered by the PET insert, a different detector concept is needed. Avalanche photodiodes (APDs) have been used in prototype small-animal PET systems [7, 8] to read out the scintillation light from bismuth germanate or cerium-doped lutetium oxyorthosilicate (LSO) crystals. APDs operate in high magnetic fields without performance degradation. In addition, these semiconductor detectors are very small, thus offering the opportunity to build very compact modules with minimum interference with MR imaging [9]. The timing resolution of APD-based detectors is in the range of a few nanoseconds, thus timeof-flight (TOF) PET is not possible with these light sensors. Promising new light sensors, silicon photomultipliers (SiPMs), offer higher gain and sub-nanosecond timing resolution [10]. SiPMs are arrays of small (e.g., $50 \times 50 \mu \mathrm{m}^{2}$ ) APDs operated beyond breakdown in Geiger counting mode. Using the summed signal of several hundred or thousand Geiger APDs, the number of hit cells and thus the number of photons hitting the matrix can be determined. Owing to the limited number of cells, the dynamic range of linear response is limited compared to the standard APDs [11], but timing performance is improved. First experiences with a PET/MR scanner based on SiPMs were recently reported with preclinical [12] and clinical [13] scanners.

\section{PET/MR system designs}

The PET/MR instrumentation available today can be one of three types: sequential systems combine both modalities in the same manner as PET/CT systems do, placing them in a tandem configuration with some space between the tomographs. A second approach relies on a removable PET insert which is placed within the bore of the MR scanner. Finally, integrated systems incorporate the PET detectors into the MR scanner.

\section{Sequential systems}

The most straightforward way to create a combined PET/MR scanner is to adapt existing PET and MR machines to work in a tandem configuration. This is the design used in clinical PET/CT scanners. In this approach, the patient is placed on a mechanical bed that slides in sequence through both scanners (Fig. 1). After reconstruction, the sensor-coded bed position information is used to perform the registration and fusion of the acquired data. An advantage of this configuration is that it minimizes adjustments of the individual components required to create the hybrid scanner.

The PET scanner can be located either in a separate room or inside the radiofrequency cage of the MR apparatus. An example of the former option is General Electric's Discovery PET/CT + MR [14]. The latter option has been adopted by Philips in their Ingenuity TF system [15].

In the case of placing both scanners in the MR cage, mutual interference and the presence of the static field have to be accounted for. This can be achieved either using magnetic field-insensitive photodetectors or by providing adequate separation and shielding of the photomultipliers. In the case of the Ingenuity $\mathrm{TF}$, the centers of the scanners are $4.2 \mathrm{~m}$ apart, the PET detector ring is surrounded by additional shielding, and each photomultiplier is inserted in a mu-metal case.
Fig. 1 Sketch of a tandem PET/ MR system. The PET scanner is positioned such that the photomultiplier-based detectors are not influenced by the magnetic field

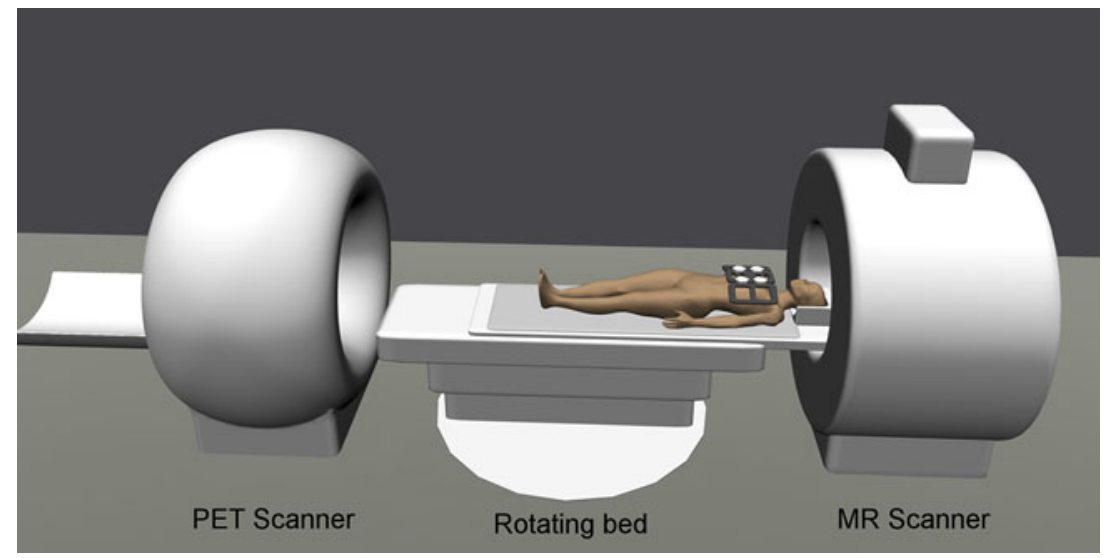


In cases in which the PET subsystem is also capable of acquiring CT data, this is of particular importance for the creation of attenuation maps, as MR-based attenuation correction is not straightforward.

The inability to perform real simultaneous acquisition of both modalities is the main limitation of this architecture. Furthermore, registration errors due to physiological activity and patient motion during the scan transition can be extremely challenging to correct.

Last but not least, tandem system configurations require larger room sizes, which might be a limitation for centers planning to update existing equipment.

\section{Insert systems}

Insert architecture was the approach used in the first research attempts to improve PET spatial resolution by exploiting the potential reduction of positron range inside a magnetic field. The idea is to build a removable insert containing a PET detector ring capable of working within the bore of a conventional MR scanner. The main technical challenge of this approach is the introduction of electronic circuits in the scanner bore, where the static field is most intense. The devices used for the scintillation light readout and signal amplification must therefore be either insensitive to the magnetic field or placed in a shielded enclosure outside the fringe field region. Furthermore, the magnetic susceptibility of all elements placed in the scanner bore must be such that the disturbance to the magnetic field is minimized. Passive shimming structures might be necessary to ensure MR image quality. Finally, electronic components in the insert must be shielded to prevent electromagnetic interference. This is particularly important in insert architectures because the PET detectors are in the field of view of the MR transmit coils. This problem is commonly avoided by designing custom transmit/receive coils to be fitted inside the PET insert.

A limitation of this architecture is the narrowing of the scanner bore due to the presence of the insert, which limits these systems to small-animal studies and either neurological or limb explorations in humans.

The same size restrictions have consequences on the performance of the PET system: limiting the radial extent of the insert means limiting the length of the scintillator crystals and thus the detector sensitivity; narrowing the detector ring leads to an increase in sensitivity and also in scatter fraction; furthermore, heat management is complicated due to the compact design.

Despite the technical challenges mentioned, insert systems allow the simultaneous acquisition of both modalities, a feature that constitutes their main advantage with respect to sequential architectures. This leads to a reduction in the total acquisition time (estimated in the order of $40 \%$ ), ensures an excellent spatial and temporal coregistration of the data, and opens the way to a range of novel applications, such as simultaneous fMR/PET, simultaneous contrast and tracer studies, MR-based motion and partial volume correction, etc.

Several working prototypes have been developed in the past for animal studies [3, 16]. APDs have been used instead of photomultiplier tubes to avoid having to guide the scintillation light outside the magnetic field. A collaboration between the University of California Davis and the University of Tubingen developed two different insert prototypes fully contained in the MR bore, one relying on short optical fiber bundles [17] and the other on direct coupling to the scintillators [18].

The first APD-based system for clinical neurology applications, the Siemens prototype BrainPET insert, proved the feasibility of performing hybrid imaging in humans [19] (Fig. 2).

Integrated systems

Although the above-mentioned architectures may offer some advantages, there is a strong interest in an approach enabling full-body scanning with simultaneous PET/MR acquisition. The way to achieve this is through complete integration of the PET detector and electronics into the MR scanner (Fig. 3). From a technical point of view, this is the most challenging approach, requiring significant changes to both subsystems.

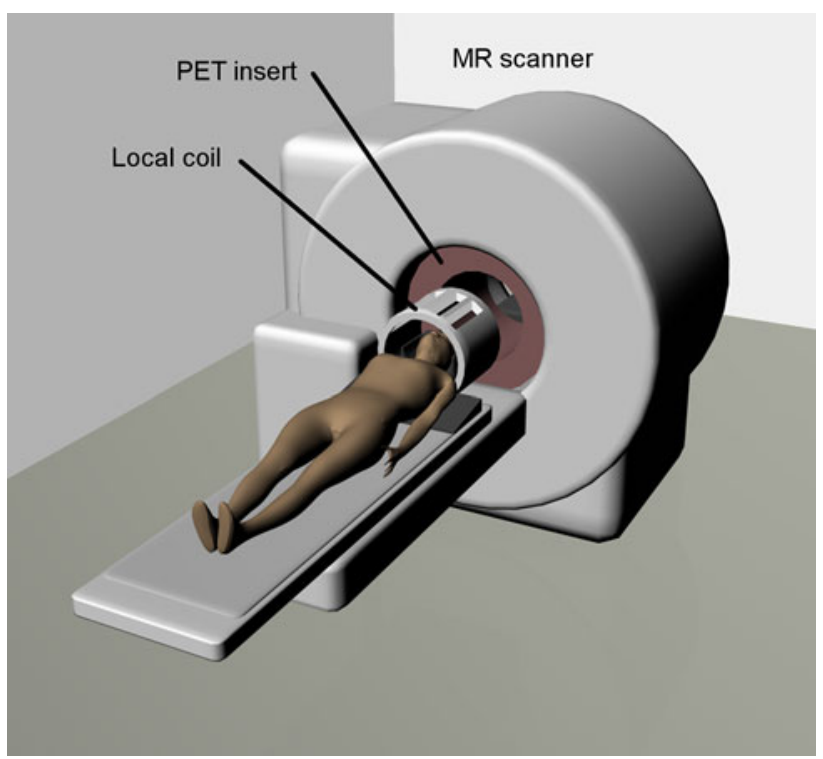

Fig. 2 Sketch of a PET insert in an MR system for simultaneous acquisition. The PET ring slides in the MR system. Owing to this geometry, the field of view is limited and does not offer whole-body clinical scans 


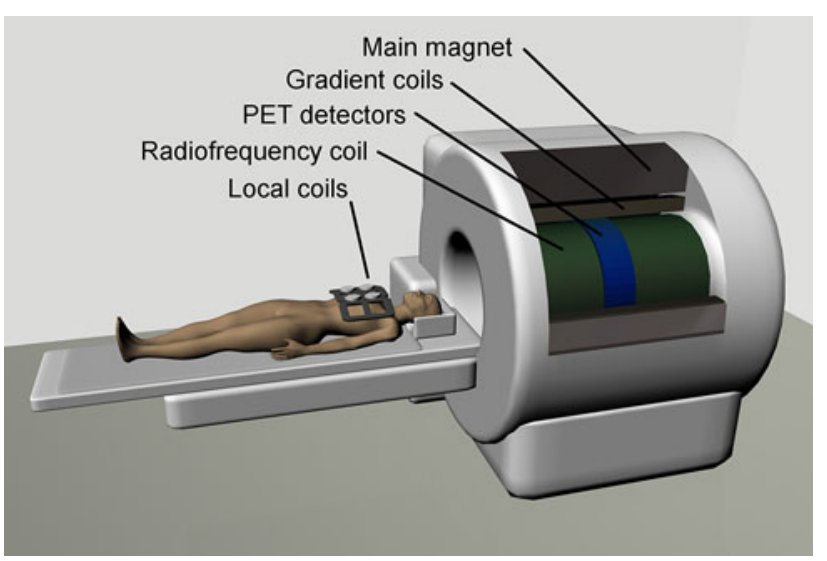

Fig. 3 Sketch of an integrated PET/MR system, offering simultaneous acquisition for human whole-body studies as well

The integrated designs published so far rely on the use of a split superconducting magnet or of field-cycled MR, or on the insertion of the PET detector ring behind the radiofrequency coil of the MR scanner.

In the first case, the MR superconducting coil is built in two separate elements, between which an axial space of several centimeters is left in which a PET scintillator ring can be accommodated. The scintillation light is guided by radially distributed fiber optic bundles to PMTs located outside the $1 \mathrm{mT}$ fringe field. Such a system was tested for preclinical imaging at the Neuroscience Department of the University of Cambridge [20]. This design requires a lowfield magnet $(\sim 1 \mathrm{~T})$ and specialized gradient set, which likely restricts this approach to small-animal imaging.

In the case of field-cycled acquisition, two separate and dynamically controllable magnets are used for polarization and readout. This makes it possible, in the acquisition of MR data, to interleave certain temporal frames free of magnetic field, in which the PET acquisition can take place. This design, like the previous one, is for the moment restricted to preclinical imaging [21].

In the last case, both the scintillator crystals and the associated photodetectors are located behind the radiofrequency coil of the MR scanner. This can be achieved either by reducing the radius of the radiofrequency coil to provide space for the PET detector or using a split gradient coil. The former is the approach adopted in the Siemens Biograph mMR [22], currently the only commercially available integrated clinical PET/MR system. The latter is the approach announced by Philips for their integrated system, presently still being developed under the EU SUBLIMA project (sub-nanosecond leverage in PET/MR imaging, http://www.sublima-pet-mr.eu/). Another significant difference between these systems is that the mMR uses APD technology, whereas Philips is aiming at TOF capability using SiPM detectors.
Fitting the detector ring between the radiofrequency and gradient coils entails problems similar to those of insert architectures: the smaller ring diameter compared to standard geometries results in better sensitivity but increases random and scattered count rates [23]. However, by reducing the energy acceptance window of the detectors, these effects can (to a certain extent) be made to cancel each other out [22].

The integration of the PET detectors behind the radiofrequency coil has the advantage of reducing the interference due to the MR excitation pulses. On the other hand, the environment temperature in that space is higher and can undergo fluctuations of tens of degrees. Since the gain of APDs and SiPMs changes with temperature, temperature stabilization by implementation of cooling close to the sensors is essential.

\section{Quantification}

PET quantification relies on accurate attenuation and scatter correction. The very short measurement time of CT, yielding attenuation information for a large body region in a few seconds, has been an important factor for the clinical success of PET/CT. With this information, scatter models have become more precise as well.

Unfortunately, the MR signal is not related to photon attenuation. Therefore, procedures have been developed and are still being refined, which assign gamma-ray attenuation factors to body regions identified on the basis of MR images. One class of algorithm is based on segmentation of patient MR data into regions of different tissue types (e.g., soft tissue, fat, lung, air) and subsequent assignment of predefined tissue attenuation values. A drawback of this method is the difficulty of identifying bone tissue in conventional MR data sets. Specific ultrashort echo time MR sequences have been established to yield information on the bones. These are successful for brain imaging, but still need to be optimized for wholebody applications [24-26].

Whole-body segmentation ignoring the bone contribution has been suggested as a practical solution [27-29]. With this method, quantification of lesions in bone is not precise [30], but for other body regions, the error is less than a few percent.

Another class of approaches is utilizing machine learning or anatomical atlases in combination with the MR data to generate realistic distributions of tissue attenuation factors. These methods coregister templates with known attenuation factors to the patient data set. Accurate matching of template and patient MR data set is required, and while robust application in brain imaging has been shown [31-33], challenges remain in whole-body 
correction. Combining the template-based and segmentation method can reduce errors introduced by non-rigid registration in the template approach [34].

Methods using the PET emission data to estimate the attenuation and activity distribution simultaneously have never been clinically successful in stand-alone PET. With the additional MR information, the results improve, and TOF PET can determine the attenuation values more accurately $[35,36]$.

Overviews of attenuation correction issues in PET/MR can be found in recent reviews [37-39].

Although more data are now available, following the introduction of combined PET/MR instrumentation for clinical use, the improvement of attenuation correction in PET/MR continues to be a topic of intensive research.

Also, scatter and attenuation due to the radiofrequency coil and any other hardware in the field of view of the PET imaging device need to be accounted for [40-42]. This can be done by implementing a known attenuation map (e.g., from CT measurements), if the position of the coils is known, or it can be determined during acquisition.

Truncation of the patient's arms in the MR field of view, occurring when the MR field of view is smaller than the PET one, is another source of inaccurate quantification and potentially strong artifacts in PET images [43]. This effect can be corrected for, on the basis of information from nonattenuation-corrected PET data [44], or it can be eliminated by extending the MR field of view [45]. Both approaches are part of ongoing research.

Measurement times for a complete multislice image or 3D dataset in MR are typically long in comparison to physiological timescales such as breathing or heartbeat. Similarly, PET measurement typically takes several minutes per bed position. In MR, several techniques have been developed to overcome this problem. Some of them rely on the capacity of MR to measure very fast navigator signals. For the PET data, this information can be used retrospectively to rebin the detected events accordingly and thus motion-freeze the data. MR-based motion compensation is a very active research field [46] and it remains to be seen which approach will be clinically successful.

Combined PET/MR technology has been available for over 2 years in the clinic. While the technical challenges have been addressed by a number of different hardware solutions, some issues remain in the area of quantification and motion compensation. Clinical data is showing image quality comparable to that of PET/CT and protocols are being developed to optimally exploit the multiparametric power of PET/MR.

Acknowledgments The combined PET/MR system at the Department of Nuclear Medicine has been funded by the Deutsche Forschungsgemeinschaft (DFG) Grossgeräteinitiative 2010.
Conflict of interest The authors declare no conflict of interest.

\section{References}

1. Hammer BE, Christensen NL, Heil BG (1994) Use of a magnetic field to increase the spatial resolution of positron emission tomography. Med Phys 21:1917-1920

2. Kraus R, Delso G, Ziegler SI (2012) Simulation study of tissuespecific positron range correction for the new Biograph mMR whole-body PET/MR system. IEEE Trans Nucl Sci 59:19001909

3. Shao Y, Cherry SR, Farahani K et al (1997) Development of a PET detector system compatible with MRI/NMR systems. IEEE Trans Nucl Sci 44:1167-1171

4. Farahani K, Slates R, Shao Y, Silverman R, Cherry S (1999) Contemporaneous positron emission tomography and MR imaging at $1.5 \mathrm{~T}$. J Magn Reson Imaging 9:497-500

5. Slates RB, Farahani K, Shao Y et al (1999) A study of artefacts in simultaneous PET and MR imaging using a prototype MR compatible PET scanner. Phys Med Biol 44:2015-2027

6. Marsden PK, Strul D, Keevil SF, Williams SC, Cash D (2002) Simultaneous PET and NMR. Br J Radiol 75 Spec No:S53-S59

7. Lecomte R, Cadorette J, Rodrigue $S$ et al (1996) Initial results from the Sherbrooke avalanche photodiode positron tomograph. IEEE Trans Nucl Sci 43:1952-1957

8. Ziegler SI, Pichler BJ, Boening G et al (2001) A prototype highresolution animal positron tomograph with avalanche photodiode arrays and LSO crystals. Eur J Nucl Med 28:136-143

9. Pichler BJ, Swann BK, Rochelle J et al (2004) Lutetium oxyorthosilicate block detector readout by avalanche photodiode arrays for high resolution animal PET. Phys Med Biol 49: 4305-4319

10. Buzhan P, Dolgoshein B, Filatov L et al (2003) Silicon photomultiplier and its possible applications. Nucl Instr Meth A 504:48-52

11. Pulko J, Schneider FR, Velroyen A, Renker D, Ziegler S (2012) A Monte-Carlo model of a SiPM coupled to a scintillating crystal. J Instrum 7:P02009

12. Yoon HS, Ko GB, Kwon SI et al (2012) Initial results of simultaneous PET/MRI experiments with an MRI-compatible silicon photomultiplier PET scanner. J Nucl Med 53:608-614

13. Ganin A, Guo J, Kim C et al (2012). Time-of-flight PET-MR detector development with silicon photomultiplier. In: PET/MR and SPECT/MR: New Paradigms. La Biodola (IT)

14. Veit-Haibach P, Kuhn FP, Wiesinger F, Delso G, von Schulthess G (2012) PET-MR imaging using a tri-modality PET/CT-MR system with a dedicated shuttle in clinical routine. MAGMA. (Epub ahead of print)

15. Zaidi H, Ojha N, Morich M et al (2011) Design and performance evaluation of a whole-body Ingenuity TF PET-MRI system. Phys Med Biol 56:3091-3106

16. Raylman RR, Majewski S, Lemieux SK et al (2006) Simultaneous MRI and PET imaging of a rat brain. Phys Med Biol 51:6371-6379

17. Catana C, Wu Y, Judenhofer MS et al (2006) Simultaneous acquisition of multislice PET and MR images: initial results with a MR-compatible PET scanner. J Nucl Med 47:1968-1976

18. Judenhofer MS, Wehrl HF, Newport DF et al (2008) Simultaneous PET-MRI: a new approach for functional and morphological imaging. Nat Med 14:459-465

19. Herzog H, Langen KJ, Weirich C et al (2011) High resolution BrainPET combined with simultaneous MRI. Nuklearmedizin 50:74-82 
20. Lucas AJ, Hawkes RC, Ansorge RE et al (2006) Development of a combined microPET-MR system. Technol Cancer Res Treat 5:337-341

21. Gilbert KM, Handler WB, Scholl TJ, Odegaard JW, Chronik BA (2006) Design of field-cycled magnetic resonance systems for small animal imaging. Phys Med Biol 51:2825-2841

22. Delso G, Furst S, Jakoby B et al (2011) Performance measurements of the Siemens mMR integrated whole-body PET/MR scanner. J Nucl Med 52:1914-1922

23. Delso G, Martinez MJ, Torres I et al (2009) Monte Carlo simulations of the count rate performance of a clinical whole-body MR/PET scanner. Med Phys 36:4126-4135

24. Keereman V, Fierens Y, Broux T et al (2010) MRI-based attenuation correction for PET/MRI using ultrashort echo time sequences. J Nucl Med 51:812-818

25. Berker Y, Franke J, Salomon A et al (2012) MRI-based attenuation correction for hybrid PET/MRI systems: a 4-class tissue segmentation technique using a combined ultrashort-echo-time/ Dixon MRI sequence. J Nucl Med 53:796-804

26. Catana C, van der Kouwe A, Benner T et al (2010) Toward implementing an MRI-based PET attenuation-correction method for neurologic studies on the MR-PET brain prototype. J Nucl Med 51:1431-1438

27. Martinez-Moller A, Souvatzoglou M, Delso G et al (2009) Tissue classification as a potential approach for attenuation correction in whole-body PET/MRI: evaluation with PET/CT data. J Nucl Med 50:520-526

28. Steinberg J, Jia G, Sammet S et al (2010) Three-region MRIbased whole-body attenuation correction for automated PET reconstruction. Nucl Med Biol 37:227-235

29. Schulz V, Torres-Espallardo I, Renisch S et al (2011) Automatic, three-segment, MR-based attenuation correction for whole-body PET/MR data. Eur J Nucl Med Mol Imaging 38:138-152

30. Keereman V, Holen RV, Mollet P, Vandenberghe S (2011) The effect of errors in segmented attenuation maps on PET quantification. Med Phys 38:6010-6019

31. Montandon ML, Zaidi H (2005) Atlas-guided non-uniform attenuation correction in cerebral 3D PET imaging. Neuroimage 25:278-286

32. Hofmann M, Steinke F, Scheel V et al (2008) MRI-based attenuation correction for PET/MRI: a novel approach combining pattern recognition and atlas registration. J Nucl Med 49: $1875-1883$

33. Malone IB, Ansorge RE, Williams GB et al (2011) Attenuation correction methods suitable for brain imaging with a PET/MRI scanner: a comparison of tissue atlas and template attenuation map approaches. J Nucl Med 52:1142-1149

34. Hofmann M, Bezrukov I, Mantlik F et al (2011) MRI-based attenuation correction for whole-body PET/MRI: quantitative evaluation of segmentation- and atlas-based methods. J Nucl Med 52:1392-1399

35. Salomon A, Goedicke A, Schweizer B, Aach T, Schulz V (2011) Simultaneous reconstruction of activity and attenuation for PET/ MR. IEEE Trans Med Imaging 30:804-813

36. Defrise M, Rezaei A, Nuyts J (2012) Time-of-flight PET data determine the attenuation sinogram up to a constant. Phys Med Biol 57:885-899

37. Bezrukov I, Mantlik F, Schmidt H, Scholkopf B, Pichler BJ (2013) MR-based PET attenuation correction for PET/MR imaging. Semin Nucl Med 43:45-59

38. Wagenknecht G, Kaiser HJ, Mottaghy FM, Herzog H. MRI for attenuation correction in PET: methods and challenges. MAGMA 2012. (Epub ahead of print)

39. Martinez-Moller A, Nekolla SG (2012) Attenuation correction for PET/MR: problems, novel approaches and practical solutions. Z Med Phys 22:299-310

40. Delso G, Martinez-Moller A, Bundschuh RA et al (2010) Evaluation of the attenuation properties of MR equipment for its use in a whole-body PET/MR scanner. Phys Med Biol 55:4361-4374

41. Tellmann L, Quick HH, Bockisch A, Herzog H, Beyer T (2011) The effect of MR surface coils on PET quantification in wholebody PET/MR: results from a pseudo-PET/MR phantom study. Med Phys 38:2795-2805

42. Paulus DH, Braun H, Aklan B, Quick HH (2012) Simultaneous PET/MR imaging: MR-based attenuation correction of local radiofrequency surface coils. Med Phys 39:4306-4315

43. Delso G, Martinez-Moller A, Bundschuh RA, Nekolla SG, Ziegler SI (2010) The effect of limited MR field of view in MR/PET attenuation correction. Med Phys 37:2804-2812

44. Nuyts J, Bal G, Kehren F et al (2012) Completion of a truncated attenuation image from the attenuated PET emission data. IEEE Trans Med Imaging 32(2):237-246

45. Blumhagen JO, Ladebeck R, Fenchel M, Scheffler K (2012) MRbased field-of-view extension in MR/PET: $\mathrm{B}(0)$ homogenization using gradient enhancement (HUGE). Magn Reson Med. doi: $10.1002 / \mathrm{mrm} .24555$

46. Ouyang J, Li Q, El Fakhri G (2013) Magnetic resonance-based motion correction for positron emission tomography imaging. Semin Nucl Med 43:60-67 